\title{
Non-Intrusive Repair of Reactive Programs
}

\author{
David Harel, Guy Katz, Assaf Marron \\ Dept. of Computer Science and Applied Mathematics \\ Weizmann Institute of Science \\ Rehovot, Israel \\ Email: firstname.lastname@weizmann.ac.il
}

\author{
Gera Weiss \\ Dept. of Computer Science \\ Ben-Gurion University of the Negev \\ Beer-Sheva, Israel \\ Email: geraw@cs.bgu.ac.il
}

\begin{abstract}
We show how, under certain conditions, programs written in the behavioral programming approach can be modified (e.g., as result of new requirements or discovered bugs) using automatically-generated code modules. Given a trace of undesired behavior, one can generate a relatively small piece of code, whose execution is interwoven at run time with the rest of the system and brings about the desired changes without modifying existing code, and without introducing new bugs. At the core of our approach is the ability of a thread of behavior to prevent the triggering of events from other threads. Our repair algorithms apply model checking to the program and transform the counterexamples produced by the model-checker into corrective modules. Our work is supported by a proof-ofconcept tool, which creates understandable modules that can be further manually managed as part of ongoing incremental system development.
\end{abstract}

Keywords-Program repair; verification; behavioral programming; model checking; patching.

\section{INTRODUCTION}

Software maintenance is a difficult and error prone task. As errors (bugs) are discovered, and requirements are added or changed, developers work hard to modify existing code without introducing new errors. They are often constrained by limited knowledge of possible side-effects, since undocumented interdependencies might be known only to a different person (usually, the original developer) who is unavailable, or have been simply forgotten. Research on automated program repair, and, more generally, program synthesis from specifications, aims to address these and related challenges. Such automation may prove particularly valuable for handling failure/bug reports from users who press the "Send to Software Vendor" button. In such cases, the software engineer cannot discuss with the user the context of the problem, or possible generalizations thereof.

In this paper we focus on repair through the forbidding of existing, faulty execution paths of programs written in the behavioral programming approach. This technique is highly suitable for (a) non-intrusive incremental repair; i.e., large parts of the system are already developed and are not modified by the repair process; (b) methodological integration of the repair process with standard, ongoing development during and after the repair activity; and (c) practical techniques for dealing with the complexity of the use of model-checking when creating local patches in the repair process.

\section{BACKGROUND}

Our work is carried out within the behavioral programming approach [9], [10] — an extension and generalization of scenario-based programming, which was introduced with the language of live sequence charts (LSCs) [4], [8], and is now implemented also in Java [10] and Erlang [11], [22].

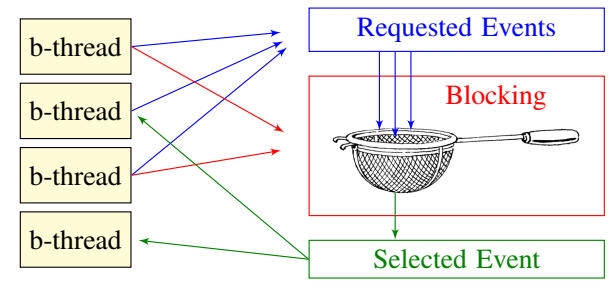

Figure 1. Behavioral programming execution cycle: all b-threads synchronize, declaring requested and blocked events; a requested event that is not blocked is selected and b-threads waiting for it are resumed.

A behavioral program consists of independent threads of behavior that are interwoven at run time. Each behavior thread (abbr. $b$-thread) specifies events and event sequences which, from its own point of view must, may, or must not occur. As shown in Fig. 1, the infrastructure synchronizes and interweaves all behaviors, selecting events that constitute integrated system behavior without requiring direct communication between b-threads. Specifically, all b-threads declare events that should be considered for triggering (called requested events) and events whose triggering they forbid (block), and then synchronize. An event selection mechanism then triggers one event that is requested and not blocked, and resumes all b-threads that requested the event. B-threads can also declare events that they simply "listenout for", and they too are resumed when these waited-for events occur.

This facilitates incremental non-intrusive development as outlined in the example of Fig. 2.

More detailed examples showing the power of incremental modularity in behavioral programming appear in [10], [11]. Briefly, in a program we wrote for playing Tic-Tac-Toe [10], 


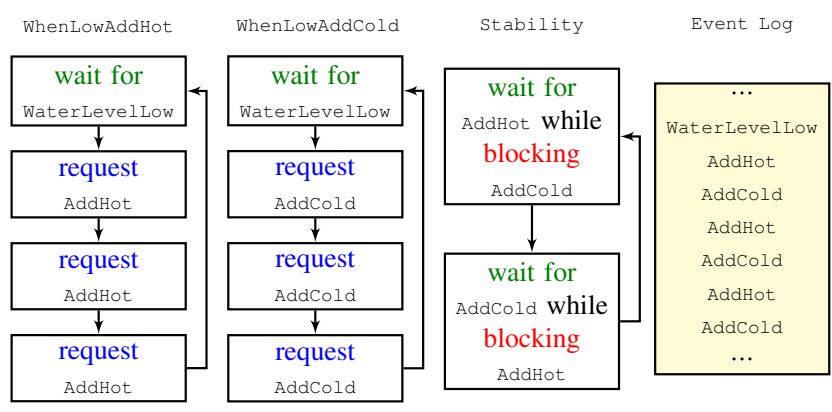

Figure 2. Incremental development of a system for controlling water level in a tank with hot and cold water sources. The b-thread WhenLowAddHot repeatedly waits for WaterLevelLow events and requests three times the event AddHot. WhenLowAddCold performs a similar action with the event AddCold, reflecting a separate requirement, which was introduced when adding three water quantities for every sensor reading proved to be insufficient. When WhenLowAddHot and WhenLowAddCold run simultaneously, with the first at a higher priority, the runs will include three consecutive AddHot events followed by three AddCold events. A new requirement is then introduced, to the effect that water temperature should be kept stable. We add the b-thread Stability, to interleave AddHot and AddCold events. For details about how sensor and actuator b-threads interact with the physical environment (sensors, valves) without suspending the entire system see [11].

each game-rule is implemented in a dedicated b-thread; e.g. "block $X$ moves when it is O's turn" or "block marking of already-marked squares”. Similarly, player-strategy modules are oblivious of other strategies; e.g., "wait for two $X$ marks in the same line, and then request marking $O$ in that line”. A similar technique can be used to control a robot performing simultaneous missions, such as vehicle operation and route management. In stabilizing a quadrotor - an unmanned flying vehicle with four rotors - each of four b-threads in our program controls a particular orientation angle, or the quadrotor's altitude, solely by changing rotor speeds; see [11].

Each b-thread repeatedly requests and blocks events representing possible increases or decreases of rotor RPM, which could contribute to its own goal. The triggering of an event that is requested by one or more b-threads and blocked by none allows at least one b-thread to progress. Affected b-threads can then recalculate their declarations of requested and blocked events, and the process repeats.

In [6] and [13], model-checking and planning algorithms (respectively) are applied to play-out, the method for executing LSCs. These smart play-out techniques control the choice of the event to be triggered, such that, within the next superstep (i.e., prior to the next event driven by the environment), the specification is not violated by the program (if this is possible). In [7], a proof-of-concept model checker verifies behavioral Java programs "in vivo" - without first translating them into a model-checker-specific language. It is further shown in [7] how, when a problem is detected, the programmer can develop and add a b-thread that repairs the program by refining the behavior without modifying existing code.

\section{OUTLINE OF THE REPAIR APPROACH}

In the present paper we utilize the model checker of [7] to automate elements of manual program-repair processes, using a principle that can be summarized as "taking the road not taken". For illustration, assume that a system was tested, or even model-checked, to satisfy its specification, and a new requirement was then introduced, or a bug reported, highlighting a required property not previously articulated, and thus neither tested nor model-checked. Our method calls for first adding the new property to the specification. We then model-check the program to find distinct violating runs. For each such run, we add a special b-thread, which waits for the sequence of all events in the run, up to the last one requested by the program (rather than by the environment). The repair b-thread then blocks this event. Some other pending requests might then be triggered. If this does not correct the problem, the process repeats.

For example, consider a faulty game-strategy b-thread, whose event request leads to a loss. When this event is blocked, another b-thread, perhaps one that requests a set of default moves, comes into play (so to speak), offering an alternative. The elimination process continues until "the right" default move is the choice at that state. The new corrective wait-and-block behavior is non-intrusive, in that its implementation does not require changing the existing program code.

We refer to such a repair b-thread as a patch, and to the process as patching, or simply, repairing. We hope that combined with the behavioral-programming principles, our approach will help make the concept of patching seem less a "necessary evil" and more a useful, mainstream software maintenance practice.

As full program repair may not always be possible, due to the state explosion problem, we also discuss the case where patching can be limited to a bounded "neighborhood" of a specific operation scenario; for example, when we are provided with a bug report sent from a user.

We formally prove correctness and analyze the method, characterize the programs on which it can be used, and exemplify its usage with our proof-of-concept tool.

The rest of this paper is organized as follows: basic definitions of behavioral programs and their model-checking are given in Sections IV and in Section V, respectively. The repair of loopless programs is discussed in Section VI, followed by a repair algorithm for general programs in Section VII. Finally, limited-depth patching is described in Section VIII. Each of the three repair algorithms is followed by a concrete example.

\section{DEFINITIONS}

While behavioral programming is geared towards natural and intuitive development using almost any programming language, its underlying infrastructure can be conveniently described and analyzed in terms of transition systems. 


\section{A. The Behavioral Programming Computational Model}

The definitions below follow [7], [11] and were modified to include the notion of a b-thread tagging states of the system as having certain properties, commonly termed atomic propositions (AP) [2]. Recall that a deterministic labeled transition system is a 6-tuple $\langle S, E, \rightarrow$, init, $A P, L\rangle$, where $S$ is a set of states, $E$ is a set of events, $\rightarrow$ is a (possibly partial) function from $S \times E$ to $S$, init $\in S$ is the initial state, $A P$ is a set of atomic propositions, and $L: S \rightarrow 2^{A P}$ is a labeling function. The runs of a transition system are sequences of the form $s_{0} \stackrel{e_{1}}{\longrightarrow} s_{1} \stackrel{e_{2}}{\longrightarrow} \cdots \stackrel{e_{i}}{\longrightarrow} s_{i} \cdots$, where $s_{0}=$ init, and for all $i=1,2, \cdots, s_{i} \in S, e_{i} \in E$, and the function $\rightarrow$ maps the pair $\left\langle s_{i-1}, e_{i}\right\rangle$ to $s_{i}$, written $s_{i-1} \stackrel{e_{i}}{\rightarrow} s_{i}$. We say that $\langle S, E, \rightarrow$, init $\rangle$ is total if the function $\rightarrow$ is total.

Behavior threads are modeled as transition systems, with $S, E$, and $A P$ finite, and the states being associated with event sets:

Definition 1. A behavior thread (abbr. b-thread) is a tuple $\langle S, E, \rightarrow$, init, $A P, L, R, B\rangle$, where $\langle S, E, \rightarrow$, init, $A P, L\rangle$ forms a deterministic total labeled transition system, $R: S \rightarrow$ $2^{E}$ associates a state with the set of events requested by the b-thread when in it, and $B: S \rightarrow 2^{E}$ associates a state with the set of events blocked by the b-thread when in it.

Definition 2. The runs of a set of b-threads $\left\{\left\langle S_{i}, E_{i}, \rightarrow_{i}\right.\right.$ , init $\left.\left.t_{i}, A P_{i}, L_{i}, R_{i}, B_{i}\right\rangle\right\}_{i=1}^{n}$ are the runs of the labeled transition system $\langle S, E, \rightarrow$, init, $A P, L\rangle$, where $S=S_{1} \times \cdots \times S_{n}$, $E=\bigcup_{i=1}^{n} E_{i}$, init $=\left\langle\right.$ init $_{1}, \ldots$, init $\left._{n}\right\rangle$, and $\rightarrow$ includes a transition $\left\langle s_{1}, \ldots, s_{n}\right\rangle \stackrel{e}{\rightarrow}\left\langle s_{1}^{\prime}, \ldots, s_{n}^{\prime}\right\rangle$ if and only if

$$
\underbrace{e \in \bigcup_{i=1}^{n} R_{i}\left(s_{i}\right)}_{e \text { is requested }} \bigwedge \underbrace{e \notin \bigcup_{i=1}^{n} B_{i}\left(s_{i}\right)}_{e \text { is not blocked }}
$$

and

$$
\bigwedge_{i=1}^{n}(\underbrace{\left(e \in E_{i} \Longrightarrow s_{i} \stackrel{e}{\rightarrow}_{i} s_{i}^{\prime}\right)}_{\begin{array}{c}
\text { affected b-threads } \\
\text { move }
\end{array}} \wedge \underbrace{\left(e \notin E_{i} \Longrightarrow s_{i}^{\prime}\right)}_{\begin{array}{c}
\text { unaffected b-threads } \\
\text { don't move }
\end{array}}) .
$$

We set $A P=\bigcup_{i=1}^{n} A P_{i}$ and, for $\left(s_{1}, \ldots, s_{n}\right) \in S_{1} \times \ldots \times S_{n}$, we define:

$$
L\left(s_{1}, \ldots, s_{n}\right)=L_{1}\left(s_{1}\right) \cup \ldots \cup L_{n}\left(s_{n}\right) .
$$

Note that when implemented in a standard programming language, we assume that b-threads do not share data, and rely solely on events for input and output. This results in the abstraction that a behavior thread is "in a state" only when synchronized with others, and that the state transition caused by executing program instructions between synchronization points is atomic.

Observe that while each b-thread is deterministic in its reaction to events, Definition 2 does not specify how events are selected, and thus there may be more than one run for a given set of $b$-threads. There could be multiple ways to select events and runs, including ones that are random, planned, or priority-based. The default behavioral execution infrastructure of LSC (in the Play-Engine and PlayGo tools), the Java package $(B P J)$ and the Erlang module $(b p)$ executes a set of b-threads based on priorities. That is, in each state of the composite system, the first event that is requested and is not blocked is selected for triggering.

Definition 3. For the transition system $T$, defined in Definition 2, a (deterministic) event selection mechanism is a function $f: S \rightarrow E$, such that for each $s \in S$ there exists a transition $s \stackrel{f(s)}{\longrightarrow} s^{\prime}$ of $T$.

Behavioral programming is designed particularly for the development of reactive systems [12], and in this context it is critical to distinguish between environment behavior and program behavior.

Definition 4. A reactive behavioral program is a set of b-threads, an event selection mechanism, and a partition of the events of the b-threads into external events representing uncontrollable occurrences coming from the environment, and internal events completely controlled by the program.

We denote the set of external events by $E_{\text {env }}$, and the set of internal events by $E_{\text {prog }}$. By convention, the patches we present in this work may block only the triggering of events in $E_{\text {prog }}$ and may not block events in $E_{\text {env }}$.

\section{B. Specifications}

We now introduce definitions that assist in the discussion of desired and undesired runs of behavioral programs.

Definition 5. For a set of b-threads $P$ and a run $\rho=$ $\left(e_{1}, e_{2}, \ldots,\right)$, such that the execution corresponding to $\rho$ is $s_{\text {init }} \stackrel{e_{1}}{\rightarrow} s_{1} \stackrel{e_{2}}{\rightarrow} s_{2} \ldots$, we define $\operatorname{APtrace}(\rho)=$ $L\left(s_{\text {init }}\right) L\left(s_{1}\right) L\left(s_{2}\right) \ldots$ and define the set of all traces of $P$ to be $\operatorname{APtraces}(P)=\{\operatorname{APtrace}(\rho) \mid \rho \in \operatorname{runs}(P)\}$.

Definition 6. A specification for a behavioral program $P$ is a linear time (LT) property $\Phi$ (i.e. a subset of $\left(2^{A P}\right)^{\omega}$ ). We say that P satisfies $\Phi$, denoted $P \vDash \Phi$, iff $A \operatorname{Ptraces}(P) \subseteq \Phi$.

Since this definition assumes infinite runs, when dealing with systems of finite runs we pad any finite run with the trace $\varnothing^{\omega}$.

It is important to note, that the same set of b-threads can satisfy $\Phi$ with one event selection mechanism, and not with another. We adopt a wider perspective here, and ensure that the patched set of b-threads satisfies $\Phi$ with all event selection mechanisms. Such patching immediately detects and fixes any bugs that could have remained hidden with a certain mechanism, but which may emerge later. An approach that takes a specific event selection mechanism into account may also be useful for some applications.

We now narrow the definition of $\Phi$, which can represent any LT property, to invariants and deadlocks. 
Definition 7. An LT $\Phi$ property over $A P$ is an invariant if there is a propositional logic formula $\varphi$ over $A P$ such that $\Phi=\left\{A_{0} A_{1} A_{2} \ldots \in\left(2^{A P}\right)^{\omega} \mid \forall j \geq 0, A_{j} \vDash \varphi\right\}$.

Intuitively, invariants are properties of the current state of the system, and do not reflect the history of events leading to it. Through invariant checking one can handle regular safety properties:

Definition 8. An LT property $\Phi$ over $A P$ is called a safety property if for all $\sigma \in\left(2^{A P}\right)^{\omega}-\Phi$ there exists a finite prefix $\bar{\sigma}$ of $\sigma$ such that

$$
\Phi \cap\left\{\sigma^{\prime} \in\left(2^{A P}\right)^{\omega} \mid \bar{\sigma} \text { is a finite prefix of } \sigma^{\prime}\right\}=\phi .
$$

Intuitively, a safety property states that no "bad" sequences of events may happen. Any run that causes such a sequence has a bad prefix; after it the run does not satisfy the property no matter how it continues.

Regular safety properties are those for which some finite automaton recognizes the bad prefixes [2], or, in our case, there is a b-thread that marks its state as bad when the bad prefix is recognized. By applying the invariant modelchecker to a program with these threads added, we can effectively handle general regular safety properties.

Definition 9. We say that a (finite) run $\rho=\left(e_{1}, e_{2}, \ldots, e_{n}\right)$ causes a deadlock if it leads to a state $s$ that has no enabled events (all requested events are also blocked).

Much like invariants, deadlocks too are properties of states in the system, and not of runs.

When patching, we will receive as input a program $P$ and an invariant $\Phi$. We will implicitly check that the system has no deadlocks; if it does, the patching algorithm will try to remove them. In particular, we will make sure that no new deadlocks are created while patching; otherwise we could "patch" a system by simply blocking all enabled events at its initial state.

\section{EXTENDING THE MODEL-CHECKING OF INVARIANTS AND DEADLOCKS}

To check that a program $P$ satisfies an invariant and does not cause a deadlock we follow the algorithm in [2], section 3.3.1, and the implementation in [7].

Any state that violates the invariant or is deadlocked is marked as "bad". We construct the state graph of the program, traverse it using DFS (trimming when arriving at a cycle), and check that all states reachable from the initial state are not bad. From each state we explore all enabled events (which reflects our decision to cater for all possible event selection mechanisms).

The runtime complexity of this algorithm, implemented as in [7], is as follows. Let $G=\left(V_{G}, E_{G}\right)$ denote the state graph constructed, and let $n$ be the number of threads and $e=|E|$ the number of events in the original program. For each state $\in V_{G}$, we have to perform $n \cdot e$ operations in order to find all its enabled events. In order to determine if a state was already visited earlier (also needed to detect cycles), assuming all states are stored in a hash table, we have to calculate a unique identifier for each state; this costs an additional $\log \left|V_{G}\right|$ operations, if we assume some optimal labeling of the states that only takes that many bits. Finally, another $\left|V_{G}\right|+\left|E_{G}\right|$ operations have to be performed to later traverse the graph. In total, we have:

$$
T_{m c}=O\left(\left|E_{G}\right|+\left|V_{G}\right| \cdot\left(n \cdot e+\log \left|V_{G}\right|\right)\right) .
$$

This complexity is the minimum price one has to pay for running a model-checker on a behavioral program. Since our technique is based on model-checking, it will necessarily be forever linked in complexity to that of model checking [2], [19], and the progress made there, for better or for worse. $T_{m c}$ thus serves a base point with which to compare the complexity of our patching algorithms, and we are interested in how much additional overhead they incur above it.

We actually use a slightly different algorithm. For our purposes, the usual model-checking that returns a single violating run does not suffice: we want to explore all runs that violate the invariant or cause a deadlock.

This is achieved as follows: we traverse the state graph using the same DFS, but whenever we reach a bad state we store that information in its predecessor states. Each state already visited in the graph will thus contain information on all its bad successors. If the state is reached again, through another route from the root, we need not traverse its subtree again: we simply update the relevant states using the data already stored (see Fig. 3).
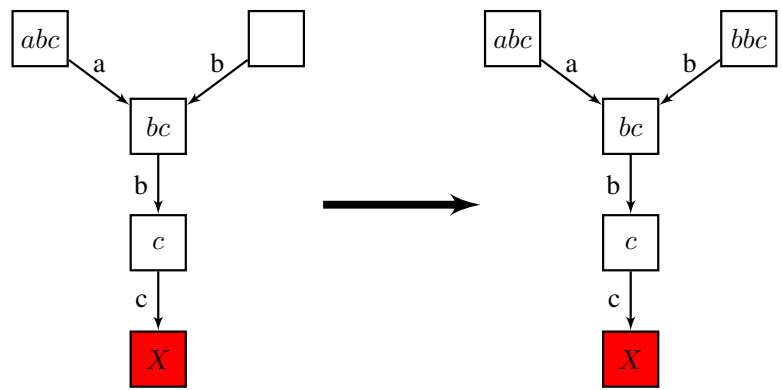

Figure 3. When a "bad" state is reached, all its predecessors store the relative path from that point to the violation. When a node in this path is reached through a different path, the data is propagated. The DFS continues until the root stores all violating paths.

The added complexity of this algorithm is measured using the number of violating runs, $\Upsilon$ (OOPSilon: pun intended), and the depth of the state graph $D$. For each violating run we propagate at most $D$ events to the predecessors, causing an overhead of $1+2+\ldots+D$ per violating run. The total runtime complexity is thus:

$$
T=T_{m c}+\Upsilon(1+2+\ldots+D)=T_{m c}+O\left(\Upsilon \cdot D^{2}\right) .
$$

Finally, if all direct successors of a state are bad, then the state itself can be considered bad; this is because the 
patching technique we discuss will cut off the violating children, rendering the state a deadlock. We thus add the following modification: if, during the DFS, all of a state's successors are violating or deadlocked, the state itself is marked as violating; thus its successors can be ignored. The runtime worst-case complexity remains unchanged.

\section{LINEAR PATCHES}

\section{A. Generating Linear Patches}

Before discussing patching of general programs, we begin with the simpler case of finite programs that are loopless: their state graph contains no cycles.

In a loopless program, every run is finite. As mentioned earlier, in such cases we add a self-looped trap state and associate it with the accepting states of the transition system.

Definition 10. A linear wait-block patch for event sequence $\left(e_{1}, e_{2}, \ldots, e_{n}, e_{\text {last }}\right)$, such that $e_{\text {last }} \in E_{\text {prog }}$, is a b-thread with the following properties:

- The patch waits for events $e_{1}, \ldots, e_{n}$, blocks $e_{\text {last }}$ once and then terminates.

- If the run deviates from the sequence $e_{1}, \ldots, e_{n}$, the patch terminates.

- The patch never requests events and does not label states $(R(s)=L(s)=\varnothing$ for all $s)$.

Intuitively, the patch is designed to prevent one bad run from occurring. Events $e_{1}, \ldots, e_{n}$ will be chosen according to violating runs found by the model-checker. The patch will intervene before the last event, causing another event to be triggered, thus preventing the violation.

The patch only interferes with runs starting with events $e_{1}, \ldots, e_{n}$; other runs remain unchanged. Formally:

Lemma 1 (The Locality Lemma). Let $P$ be a collection of b-threads, let $p$ be a linear wait-block patch for event sequence $\left(e_{1}, \ldots, e_{n}\right)$, and let $P^{\prime}=P \cup\{p\}$ denote the patched program. Then for any run $\rho$ of $P$ that does not start with events $e_{1}, \ldots, e_{n}$, the events of $\rho$ constitute a valid run $\rho^{\prime}$ of $P^{\prime}$, and APtrace $(\rho)=A \operatorname{Ptrace}\left(\rho^{\prime}\right)$.

For more details and the proof, see supplemental material available at http://www.wisdom.weizmann.ac.il/ amarron/.

The Locality Lemma is our motivation for patching: it states (in this case, for linear patches) that when we add a patch to negate a single bad run, other runs remain unharmed, meaning that the patch is local. This is an advantage of our method as compared to traditional, manual, patching: our patches do not create new errors in unexpected parts of the code.

The distinct bad runs representing the bug or emanating from the new requirement are found by model-checking:

Linear Patching $(P, \Phi)$ :

Run the model checker on $(P, \Phi)$

if $P \vDash \Phi$ then

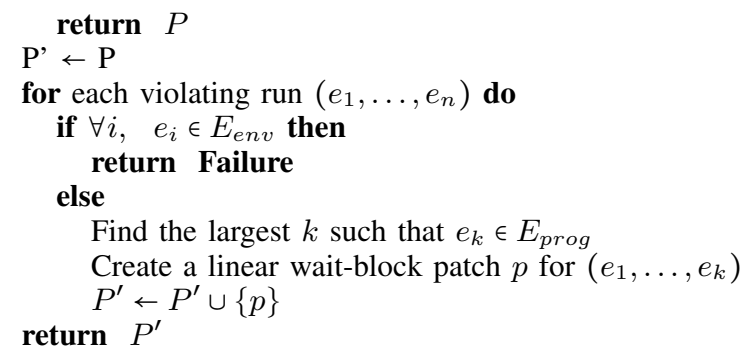

The idea is straightforward: the model-checker finds all runs violating $\Phi$ and we add a patch per run to prevent them. The algorithm guarantees that the blocking performed by the patches creates no deadlocks, by first recursively marking as "bad" any state that has only "bad" children. Furthermore, because the model-checker works with respect to all possible event selection mechanisms, any bugs that emerged after the patching are fixed. The Locality Lemma guarantees that no good runs "far away" from the patch are harmed. If the algorithm returns a patched program, we thus know that it satisfies the specification $\Phi$ and causes no deadlocks.

There is also the case where the algorithm returns a failure notice, as a result of the model checker returning a violating run in which there were no program-requested events. This, of course, means that the program cannot be repaired through wait-block patching. Formally:

Lemma 2 (The Patchability Lemma). Let $P$ be a loopless program with state graph $G=\left(V_{G}, E_{G}\right)$ and let $\Phi$ be a safety property. Then the following three statements are equivalent:

1) The algorithm succeeds in returning a patched program $P^{\prime}$.

2) There exist linear wait-block patches $p_{1}, \ldots, p_{k}$, such that $P \cup\left\{p_{i}\right\} \vDash \Phi$.

3) There exists a graph $G^{\prime}=\left(V_{G}, E_{G^{\prime}}\right)$ with $E_{G^{\prime}} \subseteq E_{G}$ and $E_{G}-E_{G^{\prime}} \subseteq E_{\text {prog }}$, such that no states violating $\Phi$ or causing deadlocks are reachable from the initial state in $G^{\prime}$.

For more details and the proof, see supplemental material available at http://www.wisdom.weizmann.ac.il/ amarron/.

Condition (3) means that the original program was "not too far" from satisfying $\Phi$ : it contained some good runs and some bad runs, and through some blocking the bad runs could be averted. Observe that the equivalence of (1) and (2) is really the validity of the algorithm.

The worst case runtime complexity of the algorithm is just that of the modified model-checker, namely $T=T_{m c}+$ $O\left(\Upsilon \cdot D^{2}\right)$. This shows the dependence of our algorithm on the number of violating runs in the original program. If their number and lengths are small enough our automatic patching is not much worse than regular model-checking. This also demonstrates why using this algorithm for synthesis could be costly. If the program is "far away" from satisfying $\Phi$, 
as could be the case when trying to synthesize a program from scratch (say, from a general program that constantly requests all possible events), then $\Upsilon$ could be polynomial in the size of the state graph, greatly slowing the process.

\section{B. Patching for a Specific Event Selection Mechanism}

The above algorithm patches the program so that it satisfies $\Phi$, regardless of the event selection mechanism used. However, it may be useful to patch the program for the specific mechanism $M$ to be used, as it could speed up the patching process, reduce the number of generated patches, and most importantly, block less events, leaving open more options for further behavior refinements and repair, as explained in Fig. 4.

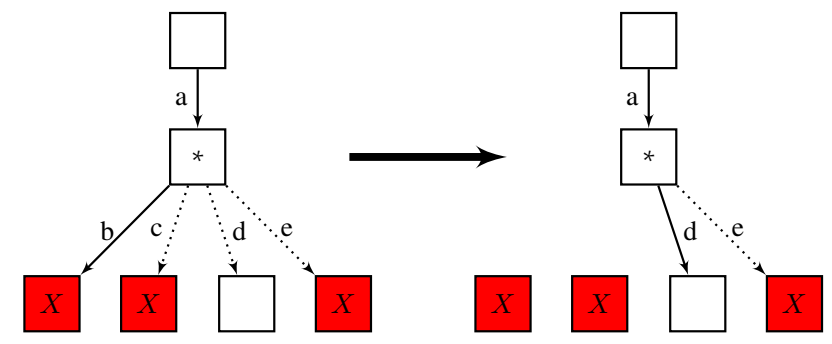

Figure 4. In state *, a patch that considers all event selection mechanisms will block $b, c$, and $e$. A patch that considers only, say, an ESM that chooses events alphabetically, needs to block $b$ and $c$, but can leave $e$ unblocked, relying on the selection of $d$.

In this case, the model-checking algorithm is modified to return as output all violating runs of the original program, as well as all (and only) violating runs that would be created by blocking previously discovered bad transitions. Bad runs that will not be possible in the patched program, under the specific ESM, are ignored. This technique is readily applicable also to patches for programs with cycles, discussed in the sequel.

\section{Example: Patching Tic-Tac-Toe}

We demonstrate the use of the linear patching algorithm on the loopless Tic-Tac-Toe behavioral program from [7]. It is loopless since the fact that each step adds a new move to the board means that its state graph has no cycles.

Suppose that the original program is developed without a model-checker. At the time of development, the programmer is convinced that the program always achieves its goal (never loses); various testers support this statement. The program is then deployed. Some months later, a customer defeats it and sends in the game's trace. However, the original software engineer has long quit the firm, and it would take a long time for a new engineer to repair the code. A suitable solution would be to apply an automatic patching algorithm to the malfunctioning software.

To simulate this, we took the complete program from [7], and omitted the more complex threads - those that handle situations where our opponent creates, simultaneously, two ways to win. If the human player does not try the complex strategy that create such double attacks, the program does indeed seem to work, but a skilled player can defeat it.

The automatic proof-of-concept tool is easy to use, requiring little modifications to the original program. The input is the behavioral program and the property $\Phi$, given as bthreads marking bad states (e.g., victory of the opponent). The output is code files for new thread instances which are easy to read and to integrate into the original program (see Fig. 5).

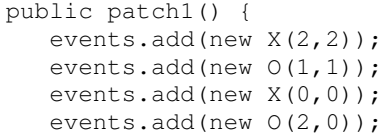

Figure 5. Example of a wait-block patch generated by the proof-of-concept tool. It waits for the moves $\mathrm{X}(2,2), \mathrm{O}(1,1), \mathrm{X}(0,0)$, and if they occur, it blocks a $\mathrm{O}(2,0)$ move. The code itself is fairly easy to comprehend; the more complicated details are hidden away in a parent class, making the auto-generated code legible and comprehensible.

Each such patch inherits from a parent class which implements its "main" function; see Fig. 6.

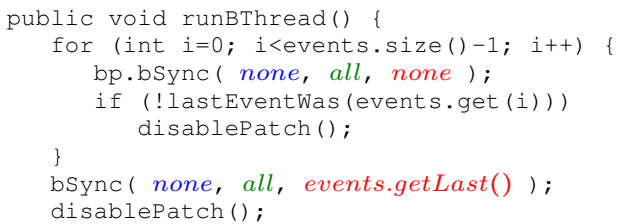

Figure 6. The patch thread's main function, runBThread () is part of the patching library, and is not added to the actual patched program. It waits for events defined by a particular patch instance (as in Fig. 5), blocking the last event and then terminating. If the events chosen deviate from those defined in the patch instance, it terminates.

In our example, the patched Tic-Tac-Toe program contains 26 different patches, one of which is demonstrated in the figure. Subsequent verification by the model checker confirms that now the specification is indeed satisfied.

\section{PATChES FOR PROGRAMS With CyClES}

\section{A. Generating Patches for Cycles}

The correctness of the algorithms for linear patching relies on the program's state graph's having no cycles. As most reactive systems run indefinitely, periodically returning to some "idle" state, such systems cannot be patched by linear wait-block patches. For example, fixing a behavioral program that enters a bad state after a sequence of events of the form $(a)^{*} b$, will call for infinitely many linear patches.

Our solution is to extend the linear patch associated with a single sequence of events, into one that can keep track of an entire hierarchy of paths and cycles in the graph, blocking the violating event as needed. 
Definition 11. Given a state graph $G^{\prime}=\left(V_{G^{\prime}}, E_{G^{\prime}}\right)$, two special vertices marked $v_{\text {init }}$ and $v_{\text {end }}$ and an event $e \epsilon$ $E_{\text {prog }}$, a cyclic wait-block patch for $G^{\prime}$ is a b-thread with the following properties:

- It waits for all events chosen by the event selection mechanism and traverses the graph $G^{\prime}$ according to those events.

- Whenever state $v_{\text {end }}$ is reached, it blocks event $e$ once.

- If an event occurs such that there is no edge marked with that event, it terminates.

- It never requests events and does not label states.

Intuitively, the patch is designed to prevent a family of bad runs that are similar to one another, in that they reach their bad state by transitioning from $v_{\text {end }}$ via the event $e$. The graph $G^{\prime}$ will be chosen such that it contains all paths from $v_{\text {init }}$ to $v_{\text {end }}$, thus rendering a single patch able to block that entire family of bad runs.

The Locality Lemma holds for the cyclic case as well: all runs of the original system, apart from those starting in $v_{\text {init }}$ and ending in reaching the violating state through $v_{\text {end }}$ and $e$, are valid runs of the patched system. The proof is based on the fact that in any such run, the generated patch does not request or block any events, and thus does not affect the events requested by the program.

Linear patches are a particular case of the cyclic ones, in which the graph $G^{\prime}$ is a path, meaning there is precisely one way to reach the violating state.

The cyclic patching algorithm is as follows $(G$ denotes the full state graph traversed by the model-checker):

Cyclic Patching $(P, \Phi)$ :

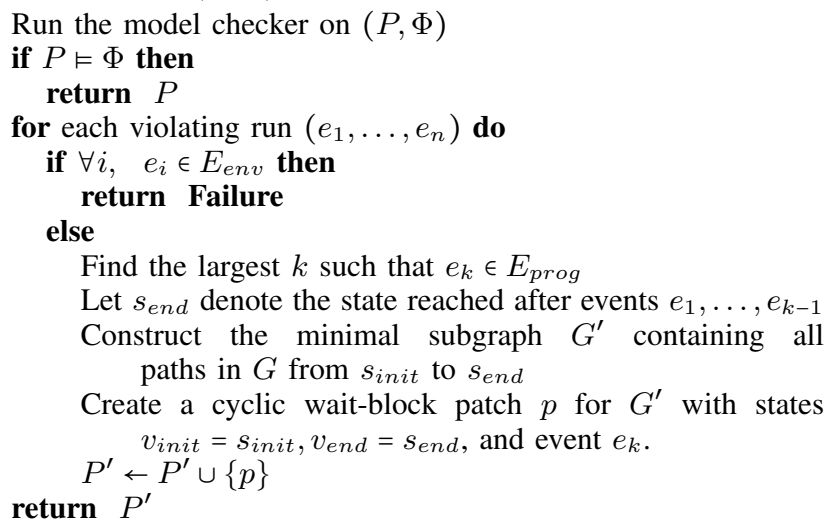

Constructing the minimal subgraph $G^{\prime}$ is performed using a modified BFS algorithm. For more details, see supplemental material at http://www.wisdom.weizmann.ac. il/ amarron/.

Lemma 3. If the algorithm returns a patched program $P^{\prime}$, then $P^{\prime} \vDash \Phi$.

Proof: Suppose that there exists a run $\rho$ of $P^{\prime}$ violating $\Phi$. Denote its states $s_{1}, \ldots, s_{n}$, and extract from them a violating run with no cycles. If $s_{i}=s_{j}$ for some $j>i$, delete states $s_{i+1}, \ldots, s_{j}$. Denote the remaining states as $s_{t_{1}}, \ldots, s_{t_{k}}$. The run corresponding to this state sequence was found by the model checker, and a patch for some subgraph $G^{\prime}$ which contains this run was created. Since $G^{\prime}$ contains all paths from $s_{1}$ to $s_{n}$, it also contains $\rho$. Therefore, the patch would have blocked the last program-requested event of $\rho$, causing a contradiction.

As with the linear case, it is possible for the algorithm to return a failure notice. The Patchability Lemma, which characterized programs that could be fixed in the linear case, holds for the cyclic case as well; its proof is analogous.

The complexity of the algorithm is as follows: The exploration of violating runs costs, as before, $O\left(T_{m c}+\Upsilon \cdot D^{2}\right)$. Constructing the relevant subgraph for each violating run costs another $\left|V_{G}\right|+\left|E_{G}\right|$ times $\Upsilon$ runs, yielding:

$$
T=O\left(T_{m c}+\Upsilon \cdot D^{2}+\Upsilon\left(\left|V_{G}\right|+\left|E_{G}\right|\right)\right) .
$$

Again, this shows our dependence on the number of violating runs, $\Upsilon$. The smaller that number, the closer our complexity is to that of the model-checker; the higher it is, the closer we are to the notorious, worst-case complexity of the synthesis problem.

\section{B. Subgraph Representation}

The generated code for a linear patch contains only the list of events to be waited for, followed by the event to be blocked. This list can be readily understood and possibly manipulated by a human, say, for documentation or analysis. Further, the developer may simplify or generalize the patch; e.g., skip waiting for certain guaranteed events or consolidate patches into fewer "symbolic" one, using BPJ's event filters. However, when a patch traverses a complex subgraph, gaining such insights is harder. Thus, we propose to represent the subgraph as a collection of easily readable linear event scenarios, amenable to human manipulation. The operation of the cyclic patch will be as before.

Specifically, We use the term line for a finite sequence of events that occur along some contiguous path in the state graph, and along which no state is visited twice. We use the term tail for a line whose last event would lead to a bad state in the state graph. The program's state graph, or parts thereof, are stored as a collection of lines, each containing its sequence of events, and links to other lines that are reachable by a single event from the last event in the line. See Fig. 7.

Thus, each patch,

- begins by activating lines containing the initial state;

- waits for all events and traverses active lines;

- deactivates active lines when they are deviated from;

- deactivates a line and activates its successors when the line's last event occurs; 


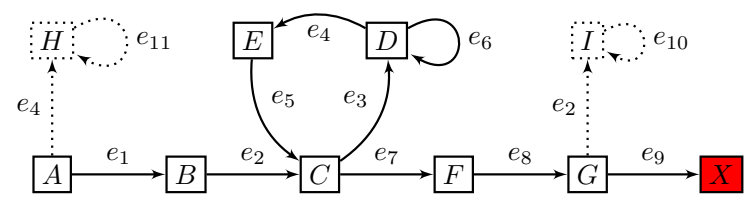

Figure 7. A state graph of a buggy program. The model-checker returns the violating run with events $e_{1}, e_{2}, e_{7}, e_{8}, e_{9}$. The subgraph of all paths from state A to state $\mathrm{G}$ (see solid states and edges) is decomposed into: line $_{1}=e_{1}, e_{2}$ (successors ${\left.\text { tail }, \text { line }_{2}\right) ; \text { line }_{2}=e_{3} \text { (successors line }}_{3}$, line 4$)$; The self-loop line $_{3}=e_{6}$ (successors line 3, line $\left._{4}\right) ;$ line $_{4}=e_{4}, e_{5}$ (successors line $_{2}$, tail) $;$ tail $=e_{7}, e_{8}$ (with event to be blocked, $e_{9}$ ). In addition to the run found by the model checker, the patch prevents other runs, e.g., $e_{1}, e_{2}, e_{3}, e_{6}, e_{6}, e_{4}, e_{5}, e_{3}, e_{4}, e_{5}, e_{7}, e_{8}, e_{9}$.

$$
\text { cycles }
$$

- in a tail, prior to the event leading to the bad state, blocks that event, waits for one more event, and deactivates the tail.

The line representation can be implemented in a data structure or in separate patch b-threads, each beginning with waiting for a unique activation event. This results in a number of small patches and is readily implementable in all implementations of behavioral programming.

\section{Example: Patching a Coffee Machine}

We demonstrate cyclic patching with a simple coffee vending machine example, which is expected to repeatedly wait for a coin, wait for a coffee request, and prepare the coffee. The main requirement is that coffee is never prepared unless a coin is first inserted. However, if immediately after power-up the user requests coffee, the machine incorrectly allows coffee to be requested and prepared infinitely many times without a coin. When the first coin is inserted, the machine enters normal operation. The machine's state graph is depicted in Fig. 8.

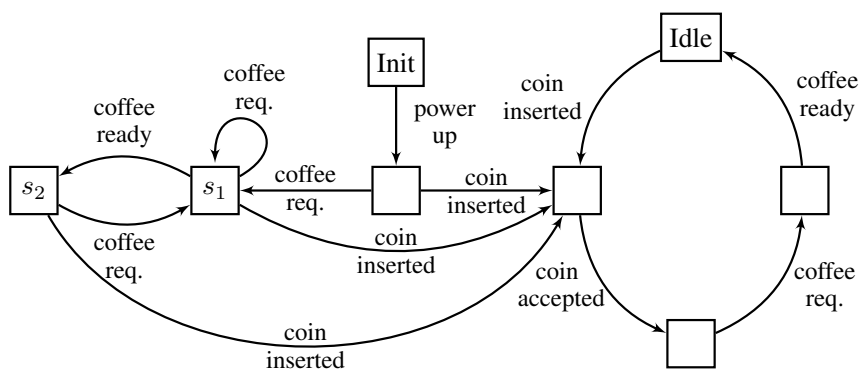

Figure 8. The buggy coffee machine's state graph. After the PowerUp event, if a CoffeeRequested event occurs (before a coin is inserted), free coffee can be obtained infinitely many times, until a coin is inserted. The loop on the right-hand side of the graph represents the desired operation. The problematic state (marked $s_{1}$ ) has two enabled events: CoffeeReady, which is immediately requested (and selected), and the environment event CoffeeRequested. We expect the patch to block the CoffeeReady event.

When the bug is discovered and automatic patching is attempted, the first step is to have a new b-thread identify and mark bad states (namely, $s_{2}$ ).
The automatic patching algorithm generates a single patch, corresponding to the subgraph depicted in Fig. 9.

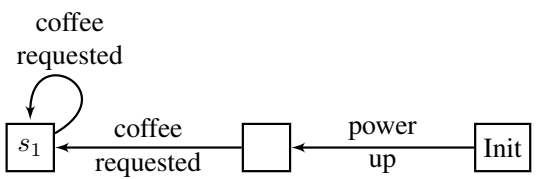

Figure 9. The subgraph of the program's state graph for which a patch is created. It shows all paths from the graph's initial state to state $s_{1}$, in which event CoffeeReady must be blocked to prevent violations.

Finally, the graph of the patched program is depicted in Fig. 10, and the code generated by the proof-of-concept tool is shown in Fig. 11.

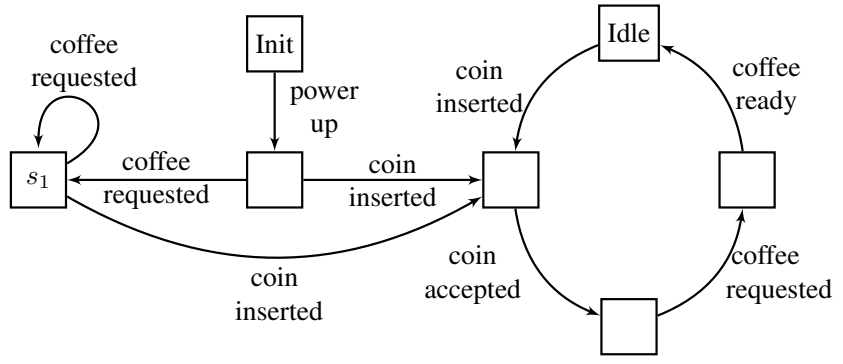

Figure 10. The patched program's state graph (states of the patches themselves are omitted for clarity). The violating CoffeeReady event has been blocked, and the bad state no longer exists in the state graph.

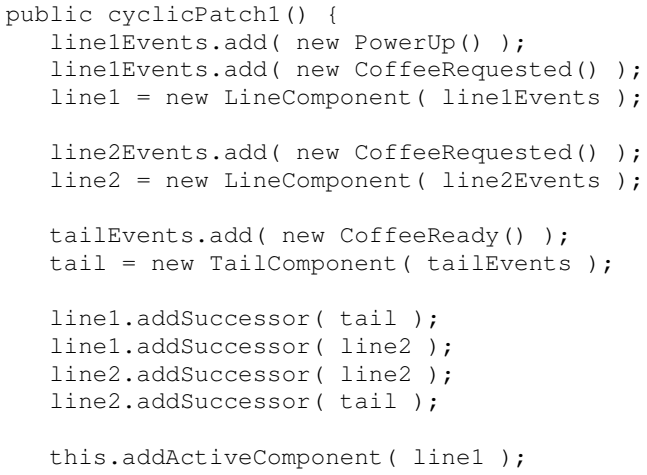

Figure 11. The automatically-generated Java code for representation of the subgraph in Fig. 9. The first line contains events PowerUp and CoffeeRequested, and the second line contains CoffeeRequested. The tail contains only the event to be blocked, CoffeeReady. The code is readily understandable.

\section{LIMITED-DEPTH REPAIR}

\section{A. Automatic Repair from Field Error Reports}

Many facilities exist for end-users to send reports of software failures to the software vendor (see, e.g., Fig. 12).

For behavioral programs, we propose a methodology for using such failure reports in order to cope with the stateexplosion problem inherent to model-checking, and to patch programs with many violating runs: 


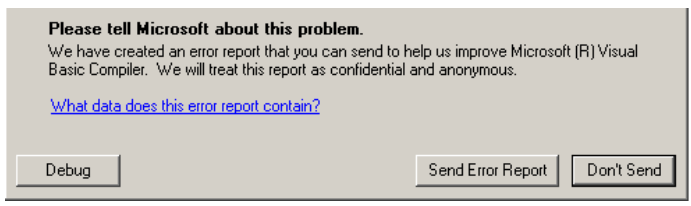

Figure 12. Event logs from bug reports are used in patch construction.

- The failure report contains an event log.

- Using the fact that the effect of a patch is local, we constrain the model checking depth to a neighborhood of the path of the failure (the bad run), followed by a limited fan-out of possible continuations, past the blocked transition.

- This is enforced by a dedicated b-thread, which monitors all events, and when an event occurs that is not along the reported bad path, it starts counting the distance from the bug report. When the distance is greater than a given parameter, the b-thread calls a model-checker API to prune the search.

- Finally, the patch is generated as above.

Such patching prevents the failure reported by the enduser, along with any other failures "not far" from it, and can help when full model-checking and patching consumes too much resources. The search-depth parameter is key, and needs to be adjusted per repaired program; higher depth means repairing more violations, but poorer performances. It is up to the user to use knowledge of the program's state graph, or run tests, in order to come up with the best choice.

\section{B. Example: Dining Philosophers}

Consider the dining philosophers problem [5]. A behavioral implementation thereof includes the events of a philosopher picking up and putting down a given fork, a b-thread for the behavior of each philosopher and a b-thread for each fork. Each philosopher's b-thread is subject to a strict event sequence: pick up one fork nondeterministically, pick up the other, put down one fork nondeterministically, and then the other. Each fork's b-thread waits for events that change the state of the fork, and blocks illegal events (e.g., a second picking up, or, a putting down by the "wrong" philosopher). In [7] we model-check this problem and variations thereof for safety and liveness properties.

The reported bug fixed is the classical deadlock where all philosophers pick up the fork on their left. The table in Fig. 13 shows the results of patching for the single bad run that we gave the patcher.

\section{RELATED WORK}

The research in [15], [17], [18] presents fault localization and automatic repair of programs, where a set of software components that are suspected to cause a fault is replaced by a set of synthesized components, such that the resulting

\begin{tabular}{|c|l|l|l|}
\hline $\begin{array}{c}\text { Search } \\
\text { Depth }\end{array}$ & 3 Philosophers & 6 Philosophers & 9 Philosophers \\
\hline \multirow{2}{*}{3} & 3 patches & 1 patches & 1 patches \\
& 3 loops & 2 loops & 2 loops \\
& 0.5 seconds & 4.2 seconds & 30 seconds \\
\hline \multirow{3}{*}{4} & 15 patches & 2 patches & 3 patches \\
& 30 loops & 4 loops & 6 loops \\
& 1.2 seconds & 22 seconds & 4.5 minutes \\
\hline \multirow{3}{*}{5} & 20 patches & 12 patches & 12 patches \\
& 380 loops & 1200 loops & 2580 loops \\
& 3.2 seconds & 2 minutes & 45 minutes \\
\hline
\end{tabular}

Figure 13. Patching the dining philosophers problem using bounded depth patching. Receiving a bug report (e.g., each philosopher picked up a single fork), the algorithm searches for event sequences that deviate from, or continue, the event trace in the bug report by no more events than the search depth parameter. The patches handle cycles discovered within the search depth (e.g., one of the philosophers completing a full cycle of picking up and putting down her two forks, while the others do not proceed). The tests were carried out on a PC with a Intel Quad Core Q6600 CPU @ 2.40GHz.

system is guaranteed to meet the full specification. Automatic repair of concurrency bugs (e.g., accessed to shared memory), is presented in [14]. The detection mechanism uses bad runs associated with bug reports, and the analysis involves actual execution. The repair is manifested in modification to existing code. Genetic-programming-based repair of legacy $\mathrm{C}$ programs is demonstrated in [21]. The repair relies on changes to existing code in order to correct problems that were assumed to be local in nature. In [1], genetic-programming is combined with co-evolution of the test cases against which the program is evaluated. Naturally, any work on automatic-repair would be considered a particular case of program synthesis [3], [16].

As for other approaches for coordinating simultaneous behaviors, such as Esterel, BIP or Linda (see related work in [9], [10] for a comparison of behavioral programming with these approaches), we believe that comparable localized repair mechanisms would be possible. The key would be implementing the equivalent of blocking which, combined with ability to subscribe to all events, is central to our solution. This, of course, is possible, as it was in Java and Erlang, and could also benefit other aspects of incremental development in these environments.

\section{Conclusion And Next Steps}

The contribution of the present paper is in the proposed automated approach, in which faulty components are neither identified nor modified. Instead, the system is nonintrusively augmented with additional components, to yield desired overall system behaviors. The entire approach is made possible by the incrementality and modularity of behavioral programs. The new components are readily understandable by humans, and can be documented, enhanced, or generalized as part of standard development. The generated patches can then be distributed to users without redistributing the original software. Finally, contributing to the on-going and up-hill battle with state explosion, we propose 
a methodology and a practical technique for constructing local patches using limited-depth model-checking.

This research is a step in the direction of developing methodologies and tools for the repair of behavioral programs. An important next step is to enrich the tool with interactive capabilities, allowing the developer to examine the state graph and enhance the proposed repairs: consolidating similar patches, generalizing or constraining patch functionality, or perhaps changing existing code after all.

Future research problems include repairing the program with regard to time-related and liveness properties and integration with other formal methods tools and techniques, including other synthesis algorithms, symbolic model-checking, and compositional verification. Our tool could be combined with Java Pathfinder [20] or other tools to explore support of richer inter-process communication beyond solely behavioral events, and possibly solving concurrency problems among b-threads, as in [14].

We hope that with further developments in incremental, non-intrusive development, supported by powerful repair automation, the task of software maintenance may eventually shed its present (often lackluster) image, becoming a rewarding undertaking, allowing software engineers to quickly address customer needs in a productive, satisfying manner.

\section{ACKNOWLEDGMENTS}

We thank A. Kantor, S. Maoz, Y. Sa'ar, S. Szekely and G. Wiener for their valuable suggestions on the manuscript, and the anonymous reviewers for their constructive comments. The research of D. Harel, G. Katz and A. Marron was supported by The John von Neumann Minerva Center for the Development of Reactive Systems at the Weizmann Institute of Science, and was partly funded by an Advanced Research Grant from the European Research Council (ERC) under the European Community's 7th Framework Programme (FP7/2007-2013). The research of G. Weiss was supported by the Lynn and William Frankel Center for CS at Ben-Gurion University and by a reintegration (IRG) grant under the European Community's FP7 Programme.

\section{REFERENCES}

[1] A. Arcuri and X. Yao. A Novel Co-evolutionary Approach to Automatic Software Bug Fixing. In Proc. 10th IEEE Congress on Evolutionary Computation (CEC), pages 162$168,2008$.

[2] C. Baier and J.-P. Katoen. Principles of Model Checking. MIT Press, 2008.

[3] R. Bloem, B. Jobstmann, N. Piterman, A. Pnueli, and Y. Saar. Synthesis of Reactive(1) Designs. Journal of Computer and System Sciences. In press.

[4] W. Damm and D. Harel. LSCs: Breathing Life into Message Sequence Charts. J. on Formal Methods in System Design, 19(1):45-80, 2001.

[5] E. W. Dijkstra. Hierarchical Ordering of Sequential Processes. Acta Inf., 1:115-138, 1971.
[6] D. Harel, H. Kugler, R. Marelly, and A. Pnueli. Smart PlayOut of Behavioral Requirements. In Proc. 4th Int. Conf. on Formal Methods in Computer-Aided Design (FMCAD), pages 378-398, 2002.

[7] D. Harel, R. Lampert, A. Marron, and G. Weiss. ModelChecking Behavioral Programs. In Proc. 11th Int. Conf. on Embedded Software (EMSOFT), pages 279-288, 2011.

[8] D. Harel and R. Marelly. Come, Let's Play: Scenario-Based Programming Using LSCs and the Play-Engine. Springer, 2003.

[9] D. Harel, A. Marron, and G. Weiss. Behavioral Programming. Communications of the ACM. To appear.

[10] D. Harel, A. Marron, and G. Weiss. Programming Coordinated Scenarios in Java. In Proc. 24th European Conf. on Object-Oriented Programming (ECOOP), pages 250-274, 2010.

[11] D. Harel, A. Marron, G. Weiss, and G. Wiener. Behavioral programming, decentralized control, and multiple time scales. In Proc. of the SPLASH Workshop on Programming Systems, Languages, and Applications based on Agents, Actors, and Decentralized Control (AGERE!), pages 171-182, 2011.

[12] D. Harel and A. Pnueli. On the Development of Reactive Systems, volume F-13 of NATO ASI Series. Springer-Verlag, New York, 1985.

[13] D. Harel and I. Segall. Planned and Traversable Play-Out: A Flexible Method for Executing Scenario-Based Programs. In Proc. 13th Int. Conf. on Tools and Algorithms for the Construction and Analysis of Systems (TACAS), pages 485499, 2007.

[14] G. Jin, L. Song, W. Zhang, S. Lu, and B. Liblit. Automated Atomicity-Violation Fixing. In Proc. ACM SIGPLAN Conf. on Programming Language Design and Implementation (PLDI), 2011.

[15] B. Jobstmann, A. Griesmayer, and R. Bloem. Program Repair as a Game. In Proc. 17th Int. Conf. on Computer Aided Verification (CAV), pages 226-238, 2005.

[16] A. Pnueli and R. Rosner. On the Synthesis of a Reactive Module. In Proc. 16th ACM Symposium Principles of Programming Languages (POPL), pages 179-190, 1989.

[17] S. Staber, B. Jobstmann, and R. Bloem. Diagnosis is Repair. In Proc. 16th Int. Workshop on Principles of Diagnosis, pages 169-174, 2005.

[18] S. Staber, B. Jobstmann, and R. Bloem. Finding and Fixing Faults. Correct Hardware Design and Verification Methods, 3275:35-49, 2005.

[19] A. Valmari. The State Explosion Problem. Lectures on Petri Nets I: Basic Models, Reisig, W. \& Rozenberg, G. (eds.), Lecture Notes in Computer Science, 1491:429-528, SpringerVerlag, 1998.

[20] W. Visser, K. Havelund, G. Brat, S. Park, and F. Lerda. Model Checking Programs. Automated Software Engineering, 10:203-232, 2003.

[21] W. Weimer, S. Forrest, C. Le Goues, and T. Nguyen. Automatic Program Repair with Evolutionary Computation. Communications of the ACM, 53:109-116, 2010.

[22] G. Wiener, G. Weiss, and A. Marron. Coordinating and Visualizing Independent Behaviors in Erlang. In Proc. 9th ACM SIGPLAN Erlang Workshop, 2010. 